\title{
GANANCIA GENÉTICA ESPERADA E INTERACCIÓN GENOTIPO-AMBIENTE EN Acacia mangium EN LA ZONA NORTE DE COSTA RICA
}

\author{
Benjamín Pavlotzky*, Olman Murillo ${ }^{1 / *}$
}

\begin{abstract}
Palabras clave: Mejoramiento genético; ensayos genéticos; interacción genotipo-ambiente; correlación genética; Acacia mangium; Costa Rica.

Keywords: Tree improvement; provenances; progeny test; genotype-environment interaction; genetic correlation; Acacia mangium; Costa Rica.
\end{abstract}

Recibido: 01/02/14

\section{RESUMEN}

Se evaluaron 2 ensayos de progenie de Acacia mangium Willd., con el objetivo de contribuir a aumentar su rentabilidad en plantaciones comerciales. Las 25 familias de polinización abierta fueron establecidas en el 2006 en los cantones de Los Chiles y San Carlos, zona norte de Costa Rica, y evaluados en 2007 y 2010. El material genético provino de selecciones de árboles plus realizadas por GENFORES, cooperativa costarricense de conservación y mejoramiento genético forestal. Cada accesión estuvo representada por 48 progenies, divididas en 4 parejas de individuos, aleatoriamente distribuidas dentro de cada uno de 6 bloques del ensayo. En la medición del 2010 se evaluó el DAP, tasa de sobrevivencia, número de piezas comerciales por árbol, presencia de bifurcación, altura de bifurcación y calidad de las primeras 4 trozas. Con base en estas mediciones, se estimó el volumen comercial por árbol y por hectárea. Los datos fueron analizados mediante el software especializado SELEGEN de EMBRAPA, Brasil, con el fin de determinar todos los parámetros genéticos de la población de mejoramiento. Todos los caracteres evaluados exhibieron valores de heredabilidad familiar

1 Autor para correspondencia. Correo electrónico: omurillo@itcr.ac.cr

* Sistema Nacional de Áreas de Conservación, San José, Costa Rica.
Aceptado: 15/07/14 
media superior $a h^{2}{ }_{\mathrm{fM}}>0,46$. La ganancia genética estimada en volumen comercial por hectárea fue de $55,8 \%$, si se selecciona como progenitores los 2 mejores individuos dentro de las mejores 12 familias del ranking, lo que corresponde a una producción en volumen comercial de $78,93 \mathrm{~m}^{3} \cdot \mathrm{ha}^{-1}$ a los 4 años (aproximadamente $20 \mathrm{~m}^{3} \cdot \mathrm{ha}^{-1} \cdot \mathrm{año}^{-1}$ ). Las 2 procedencias colombianas exhibieron un crecimiento significativamente superior a todo el resto de materiales evaluados. No se observó interacción genotipo-ambiente significativa entre ambos sitios. Las correlaciones genéticas entre los caracteres evaluados mostraron que la tasa de crecimiento del DAP se expresa tempranamente en esta especie, y por tanto, podría ser utilizada en una reducción a futuro del proceso de selección.

\section{INTRODUCCIÓN}

La acacia (A. mangium) ha sido conocida por su capacidad de crecimiento y alta producción de biomasa en suelos degradados, ácidos, de baja fertilidad y poco contenido de materia orgánica (Nirsatmanto et ál. 2004, Kim et ál. 2008). Algunas especies del género Acacia, perteneciente a la familia Fabaceae, han tenido éxito en los programas de reforestación comercial en la zona oriental del trópico asiático. En Vietnam, Van Bueren (2004) estima una superficie de 127000 ha plantadas con híbridos del género Acacia, mientras que Kim et ál. (2008), estiman en 500000 ha plantadas con A. mangium, A. auriculiformis y el híbrido de ambas. En Indonesia Nirsatmanto et ál. (2004) estiman 800000 ha plantadas con $A$. mangium, lo que refleja que en el trópico asiático esta especie se ha convertido en la opción de plantación que genera la mayor productividad en ciclos cortos de crecimiento. En Brasil se ha reportado la introducción de varias especies de este género, que incluyen la Acacia mangium, para fines de producción de carbón, como fijadora de nitrógeno y en especial, en recuperación de sitios impactados por minería (Caproni et ál. 2005, Balieiro et estimated as $55.8 \%$, when selecting as parents the 2 best individuals from the top 12 families, which would correspond to an expected commercial volume.ha- ${ }^{-1}$ at 4 years age, of $78.93 \mathrm{~m}^{3} \cdot \mathrm{ha}^{-1}$ (around $20 \mathrm{~m}^{3} \cdot \mathrm{ha}^{-1} \cdot \mathrm{year}^{-1}$ ). The 2 Colombian provenances were significantly superior in growth to the rest of the evaluated materials. No significant gene-environment interaction was observed between both sites. Genetic correlations among evaluated traits showed that diameter growth rate is expressed early in this tree species, thus could be used in shortening future selections.

ál. 2007, De Assis et ál. 2011). En Costa Rica, esta especie fue introducida en los 80, como parte de las investigaciones para la producción de leña y energía en Centroamérica (Mesén 1990, Salazar y Mesén 1990, CATIE 1992). No obstante su gran potencial, es una especie que ha recibido poca atención por parte de los silvicultores nacionales (Guevara y Murillo 2009).

Como parte del manejo exitoso de esta especie en plantaciones comerciales, es necesaria la selección y desarrollo de individuos que muestren superioridad genética en calidad del fuste, tasa de crecimiento y calidad de la madera. Trabajos en mejoramiento genético iniciados en la costa caribe colombiana, reportan estimados de progreso genético de un $30 \%$ en altura comercial, un $60 \%$ en volumen comercial y aproximadamente un $28 \%$ en calidad del fuste (Espitia et ál. 2010). En Costa Rica y en Colombia se inició recientemente un programa conjunto de mejoramiento genético con esta especie, apoyado en la cooperativa de mejoramiento genético (GENFORES) y con la participación de varias empresas reforestadoras (Espitia et ál. 2010, Pastrana 2011, Pavlotkzky y Murillo 2012, Pastrana et ál. 2012). 
Esta investigación se realizó con el objetivo de determinar diferencias genéticas y de interacción genotipo-ambiente entre procedencias y progenies de $A$. mangium en 2 regiones al norte de Costa Rica. De esta forma se espera seleccionar el mejor material genético para el programa, en relación con su tasa de crecimiento, calidad de fuste y adaptabilidad a suelos ácidos, pobres y de menor productividad (Murillo y Badilla 2004a).

\section{MATERIALES Y MÉTODOS}

Durante el 2006 se estableció un ensayo de progenie de 25 familias de A. mangium en el cantón de Los Chiles y otro ensayo idéntico en el cantón de San Carlos, ambos en la provincia de Alajuela, Costa Rica. Se utilizaron progenies provenientes de árboles plus de Colombia (códigos $\mathrm{K}=$ Kanguroid y $\mathrm{M}=$ Córdoba) y Costa Rica (códigos $\mathrm{P}=$ Parrita y $\mathrm{C}=\mathrm{El}$ Concho, San Carlos), así como 2 lotes mezclados provenientes de República Dominicana (RDom) y Papúa Nueva Guinea respectivamente. Todas las familias fueron obtenidas de polinización abierta (medios hermanos). Los ensayos forman parte del primer programa de mejoramiento genético de A. mangium en Costa Rica y Colombia, llevado a cabo por la cooperativa GENFORES.

El diseño experimental de los ensayos genéticos es el empleado y propuesto por GENFORES (Murillo y Badilla 2004b, Murillo 2011). Consiste en un diseño de bloques completos al azar con 6 bloques. Dentro de cada bloque se distribuyen 4 parejas de medios hermanos aleatoriamente y distantes entre sí, con el objetivo posterior de convertirse en huerto semillero. Los árboles fueron plantados con un distanciamiento de $3 \mathrm{~m}$ x $3 \mathrm{~m}$. El terreno fue previamente subsolado a $30 \mathrm{~cm}$ de profundidad sobre las líneas de siembra, y posteriormente rastreado.

Ambos ensayos fueron establecidos en sitios que pertenecen a la zona de vida Bosque Húmedo Tropical transición a Perhúmedo. Los datos climatológicos de la región (SIRZEE 2010, basados en el Instituto Meteorológico Nacional) reportan una precipitación promedio de
2500-3000 mm.año-1 con un periodo seco de 2 a 3 meses, el brillo solar es de 1825 horas por año, mientras que la temperatura promedio anual es de $25,3^{\circ} \mathrm{C}$ y la velocidad máxima del viento de $31 \mathrm{~km} / \mathrm{h}$. El orden de suelos de esta región norte es Ultisol y la calidad de sitio en crecimiento reportada en Los Chiles es Media y en San Carlos Media-Baja (Murillo y Badilla 2006).

Los caracteres evaluados a los 4 años fueron a) el diámetro a 1,3 m (DAP), b) el incremento del DAP en 3 años, en relación con la medición del 2007; c) presencia o ausencia de bifurcación (pérdida del eje principal); d) número de tora donde aparece la primera bifurcación (altura de bifurcación); e) sobrevivencia; f) calidad del árbol para producción de madera sólida (en una escala de $0 \%$ a $100 \%$ ), basado en la calidad individual de las primeras 4 trozas (T1, T2, T3 y T4) de 2,5 m de longitud, según la metodología propuesta por Murillo y Badilla (2004b), donde Calidad del árbol $=\mathrm{T} 1 * 0,4+\mathrm{T} 2 * 0,3+\mathrm{T} 3 * 0,2+\mathrm{T} 1 * 0,1$, para obtener un promedio ponderado por árbol; g) volumen comercial sin corteza por árbol (a una altura comercial hasta los $10 \mathrm{~cm}$ de diámetro o hasta una altura menor si se ha perdido la dominancia apical) calculado con el programa Avalúos Forestales que utiliza un modelo de estimación del volumen para Acacia mangium (Murillo y Badilla 2011); h) volumen comercial por hectárea a los 4 años, que se estimó a partir del volumen comercial individual y proyectado en una hectárea con 1111 árboles, ajustado por la mortalidad promedio registrada de su familia respectiva.

El análisis genético de los datos se realizó con los procedimientos de Máxima Verosimilitud Restringida (Restricted Maximum Likelihood, REML) y Mejor Predicción Linear No Sesgada (Best Linear Unbiased Prediction, BLUP), con el software SELEGEN (Resende 2007), y el modelo 4 (bloques al azar, progenies de medios hermanos, varias plantas por parcela, varias localidades) y el 14 (bloques al azar, varias poblaciones o procedencias, progenies de medios hermanos, varias localidades): 
Modelo 4: $\mathrm{y}=\mathrm{Xr}+\mathrm{Za}+\mathrm{Wp}+\mathrm{Ti}+\mathrm{e}$

Modelo 14: $\mathrm{y}=\mathrm{Xr}+\mathrm{Za}+\mathrm{Wp}+\mathrm{Qs}+\mathrm{Ti}+\mathrm{e}$

Donde "y" es el vector de datos, "r" es el vector de los efectos de la repetición sumados a la media general, "a" es el vector de los efectos genéticos aditivos individuales, "p" es el vector de los efectos de la parcela (todos los individuos de una familia en un bloque), "s" es el vector de los efectos poblacionales, "i" es el vector de los efectos de la interacción genotipo x ambiente, "e" es el vector de errores residuales. Las letras mayúsculas representan las matrices de incidencia para los efectos referidos (Resende 2006).

También se utilizó el modelo 106 de SELEGEN (Optimización de la selección en función de la endogamia y del tamaño efectivo poblacional), para seleccionar los mejores 2 individuos de las mejores familias del ranking genético para el volumen comercial. Con esto se pretendió obtener una ganancia genética alta, pero con la mayor diversidad genética posible en la población de mejoramiento.

La correlación genética entre caracteres se estimó después de eliminados los efectos ambientales (ecuación 3), con base en el software SELEGEN (Resende 2007). La significancia de los valores de correlación fue evaluada con la prueba estadística de " $\mathrm{t}$ " (ecuación 4), según el procedimiento sugerido por Steel y Torrie (1980):

$$
r_{a(x, y)}=\frac{\operatorname{cov}_{\hat{\mathrm{a}}(x, y)}}{\hat{\sigma}_{\hat{\mathrm{a}} x} \hat{\sigma}_{\hat{\mathrm{a}} y}}
$$

Donde, $\operatorname{cov}_{\hat{\mathrm{a}}(x, y)}$ es la covarianza genética entre los caracteres "X" and "Y", mientras que $\hat{\sigma}_{\hat{a} \mathrm{x}} \mathrm{y} \hat{\sigma}_{\hat{a} y}$ son las desviaciones estándar genéticas de los caracteres " $X$ " y "Y" respectivamente.

$$
t=\frac{r_{a(x, y)}}{\sqrt{\frac{1-r_{a(x, y)}^{2}}{n-2}}}
$$

El tamaño efectivo de población se determinó a partir de la función sugerida por Vencovsky (Resende 2002):

$$
\mathrm{Ne}=\frac{4 * \mathrm{Nf} * \mathrm{kf}}{\mathrm{Kf}+3}
$$

Donde, $\mathrm{Nf}$ es el número de familias seleccionadas; mientras que $\mathrm{kf}$ es el número promedio de individuos seleccionados dentro de familias. Ambos parámetros de la función aumentan con cada nuevo individuo incluido en la selección, en particular si el árbol pertenece a una nueva familia.

\section{RESULTADOS}

En el Cuadro 1 se muestran los parámetros genéticos de cada uno de los 8 caracteres investigados, en la evaluación de las 25 familias de Acacia mangium en 2 regiones de la zona norte de Costa Rica.

Los 8 caracteres evaluados registraron valores altos de varianza genética aditiva (Va), así como de heredabilidad media familiar $\left(\mathrm{h}^{2} \mathrm{mf}\right)$, que oscilaron entre $46 \%$ y $94 \%$, lo que indica un alto control genético y potencial importante para mejoramiento genético. La exactitud fue también muy alta en todos los caracteres, donde el menor valor fue de un $68 \%$.

El crecimiento medio en general para la especie es relativamente alto, a pesar de ser una región con condiciones de suelo sumamente pobres. El DAP registró en promedio un crecimiento cercano a los $4 \mathrm{~cm} \cdot a n \mathrm{n}^{-1}$, mientras que el IMA del volumen comercial fue de cerca de $11 \mathrm{~m}^{3} \cdot \mathrm{ha}^{-1} \cdot \mathrm{año}^{-1}$ a pesar de la corta edad. La calidad del fuste en general fue relativamente baja, 
Cuadro 1. Parámetros genéticos de familias de medios hermanos de A. mangium a los 4 años, zona norte, Costa Rica.

\begin{tabular}{|c|c|c|c|c|c|c|c|c|}
\hline Parámetros & $\begin{array}{l}\text { Volumen } \\
\text { comercial.ha }\end{array}$ & $\begin{array}{c}\text { Volumen } \\
\text { comercial.árbol }^{-1}\end{array}$ & Calidad & $\begin{array}{c}\text { DAP } \\
\text { año 1 } \\
(\mathrm{cm})\end{array}$ & $\begin{array}{c}\text { DAP } \\
\text { año } 4 \\
(\mathrm{~cm})\end{array}$ & $\begin{array}{c}\text { Incremento } \\
\text { anual del } \\
\text { DAP } \\
\left(\mathrm{cm} \cdot \tilde{a n ̃ o}^{-1}\right)\end{array}$ & Bifurcación & $\begin{array}{l}\text { Altura } \\
\text { bifurcación } \\
\text { (m) }\end{array}$ \\
\hline Va & 385,21 & 0,0003 & 67,49 & 0,67 & 5,91 & 0,23 & 0,01 & 0,09 \\
\hline Vint & 14,00 & 0,0000 & 1,42 & 0,06 & 0,06 & 0,03 & 0,001 & 0,01 \\
\hline $\mathrm{Ve}$ & 236,47 & 0,0003 & 290,88 & 0,84 & 1,7 & 1,08 & 0,14 & 1,04 \\
\hline Vf & 647,24 & 0,0006 & 367,09 & 1,58 & 7,76 & 1,35 & 0,15 & 1,17 \\
\hline $\mathrm{h}^{2} \mathrm{a}$ & $0,59 \pm 0,13$ & $0,51 \pm 0,12$ & $0,18 \pm 0,07$ & $0,42 \pm 0,11$ & $0,76 \pm 0,14$ & $0,17 \pm 0,07$ & $0,05 \pm 0,03$ & $0,07 \pm 0,04$ \\
\hline$c^{2}$ int & 0,02 & 0,001 & 0,004 & 0,04 & 0,01 & 0,02 & 0,01 & 0,005 \\
\hline $\mathrm{h}^{2} \mathrm{mf}$ & 0,88 & 0,93 & 0,79 & 0,79 & 0,94 & 0,68 & 0,46 & 0,57 \\
\hline Exactitud & 0,94 & 0,97 & 0,89 & 0,89 & 0,97 & 0,83 & 0,68 & 0,76 \\
\hline$h^{2} \mathrm{ad}$ & 0,55 & 0,45 & 0,15 & 0,38 & 0,72 & 0,14 & 0,04 & 0,06 \\
\hline rgloc & 0,87 & 0,99 & 0,92 & 0,73 & 0,96 & 0,67 & 0,62 & 0,8 \\
\hline SEP & 3,36 & 0,0023 & 1,88 & 0,19 & 0,3 & 0,13 & 0,03 & 0,1 \\
\hline $\begin{array}{l}\text { Promedio } \\
\text { general }\end{array}$ & 43,08 & 0,04 & 54,4 & 3,26 & 15,41 & 3,8 & 0,8 & 3,37 \\
\hline
\end{tabular}

Donde:

Va: varianza genética aditiva.

Vint: varianza de la interacción genotipo - ambiente.

Ve: varianza residual.

Vf: Va + Vint + Vparc + Ve: varianza fenotípica total. No se incluyó la Vparc por registrar valores sumamente bajos.

$\mathbf{h}^{2} \mathbf{a}$ : heredabilidad individual en sentido estricto, es decir, de los efectos aditivos.

$\mathbf{c}^{2}$ int: coeficiente de determinación de los efectos de interacción genotipo-ambiente.

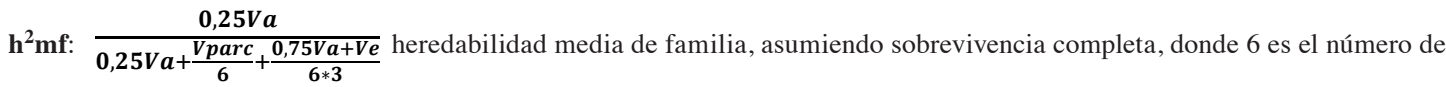
repeticiones (o bloques) y 3 el número de individuos/familia/bloque (parcela) antes del raleo.

Exactitud: raíz cuadrada de $\mathrm{h}^{2} \mathrm{mf}$ : exactitud de selección de familias, asumiendo sobrevivencia completa.

$\mathbf{h}^{2} \mathbf{a d}: \frac{\mathbf{0 , 7 5 V a}}{\mathbf{0 , 7 5 V a}+\boldsymbol{V} \boldsymbol{e}}$ heredabilidad aditiva dentro de parcelas.

Rgloc: correlación genotípica entre el desempeño de las familias en varios ambientes (estimación de la interacción genotipo-ambiente).

SEP: desviación estándar del valor genotípico estimado de familia, asumiendo sobrevivencia completa.

Promedio general del experimento para cada uno de los caracteres evaluados.

con valores de poco más de un 54\%, en una escala de 1 a 100\% (Murillo y Badilla 2004b).

En el Cuadro 2 se presentan los resultados de las correlaciones genéticas entre los caracteres investigados.

El volumen comercial individual y el volumen comercial por hectárea se correlacionaron en gran medida con el Incremento en DAP $\left(\mathrm{r}=0,79^{* *}\right)$ como se espera, pero no así con los caracteres cualitativos (calidad, bifurcación, altura de bifurcación). El volumen comercial por hectárea y la calidad registraron una correlación débil pero inversa $(\mathrm{r}=-0,28)$, mientras que la calidad del árbol correlacionó inversamente proporcional con el incremento en diámetro $\left(\mathrm{r}=-0,54^{* *}\right)$. El DAP al año 1 registró una correlación negativa de $\mathrm{r}=-0,30$ con la calidad, que se acentuó al año 4 donde aumentó a $\mathrm{r}=-0,50 * *$. Como se espera, todos los caracteres cuantitativos estuvieron positivamente correlacionados entre sí. 
Cuadro 2. Matriz de correlaciones genéticas de los caracteres investigados en familias de medios hermanos de A. mangium a los 4 años, en la zona norte de Costa Rica.

\begin{tabular}{|c|c|c|c|c|c|c|c|c|}
\hline Caracter & $\begin{array}{c}\text { Volumen } \\
\text { comercial.ha- }{ }^{-1} \\
\left(\mathrm{~m}^{3}\right)\end{array}$ & $\begin{array}{l}\text { Volumen } \\
\text { comercial. } \\
\text { árbol }^{-1}\left(\mathrm{~m}^{3}\right)\end{array}$ & Calidad & $\begin{array}{l}\text { DAP } \\
\text { año } 1\end{array}$ & $\begin{array}{l}\text { DAP } \\
\text { año } 4\end{array}$ & $\begin{array}{c}\text { Incremento } \\
\text { del DAP } \\
\text { años } 1 \text { al } 4\end{array}$ & Bifurcación & $\begin{array}{c}\text { Altura } \\
\text { bifurcación }\end{array}$ \\
\hline Volumen comercial.ha ${ }^{-1}$ & 1,00 & $0,98 * * *$ & $-0,28$ & $0,87 * *$ & $0,95 * * *$ & $0,79 * *$ & 0,12 & 0,05 \\
\hline Volumen comercial.árbol $^{-1}$ & 0,98 & 1,00 & $-0,25$ & $0,87 * *$ & $0,95 * * *$ & $0,80 * *$ & 0,11 & 0,08 \\
\hline Calidad & $-0,28$ & $-0,25$ & 1,00 & $-0,30$ & $-0,50^{*}$ & $-0,54^{*}$ & $-0,68 * *$ & $0,87 * *$ \\
\hline DAP año 1 & $0,87 * *$ & $0,87 * *$ & $-0,30$ & 1,00 & $0,90 * * *$ & $0,76 * *$ & $-0,03$ & 0,06 \\
\hline DAP año 4 & $0,95 * * *$ & $0,95^{* * *}$ & $-0,50^{*}$ & $0,90 * * *$ & 1,00 & $0,89 * * *$ & 0,25 & $-0,17$ \\
\hline Incremento DAP años 1-4 & $0,79 * *$ & $0,80 * *$ & $-0,54^{*}$ & 0,76 & 0,89 & 1,00 & 0,37 & $-0,30$ \\
\hline Bifurcación & 0,12 & 0,11 & $-0,68^{*}$ & $-0,03$ & 0,25 & 0,37 & 1,00 & $-0,83 * *$ \\
\hline $\begin{array}{l}\text { Altura } \\
\text { bifurcación }\end{array}$ & 0,05 & 0,08 & $0,87 * *$ & 0,06 & $-0,17$ & $-0,30$ & $-0,83^{* *}$ & 1,00 \\
\hline
\end{tabular}

4. La significancia de la correlación corresponde a ns=no significativo; $*=\mathrm{p}<0,05 ; * * \mathrm{p}<0,01 ; * * *=\mathrm{p}<0,001$.

La variable "supervivencia" no presentó evidencia de un control genético importante. En promedio los datos indican que un $8 \%$ de los individuos del ensayo se encontraban muertos al momento de la medición a los 4 años.

El cálculo de la variable "volumen comercial" por hectárea se realizó por medio de integración de todas las variables de calidad, crecimiento y supervivencia. Es, por lo tanto, un indicador del valor económico real del árbol que puede ser analizado genéticamente y permite determinar el mejor material genético de acuerdo con los objetivos de la plantación: obtener la mayor cantidad de madera de la mejor calidad posible con turnos de corta reducidos y con la menor inversión en mantenimientos y replantes (Figura 1).

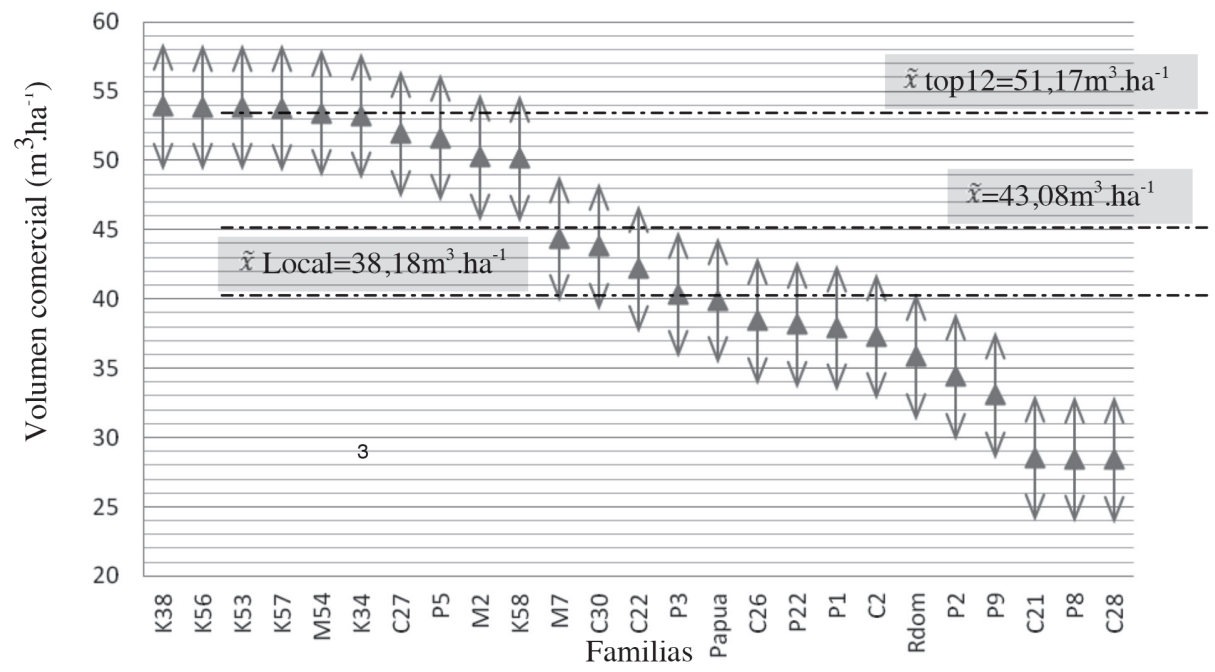

Fig. 1. Valores genéticos y límites de confianza para el volumen comercial por hectarea posterior al raleo, en un ensayo de progenies de A. mangium a los 4 años, en la zona norte de Costa Rica. 
Las mejores 11 familias en volumen comercial no registraron diferencias significativas entre sí. El promedio de las mejores 12 familias fue de $51,17 \mathrm{~m}^{3}$.ha-1, el diferencial de selección correspondiente fue de $8,09 \mathrm{~m}^{3} \cdot \mathrm{ha}^{-1} \mathrm{y}$ la heredabilidad media familiar $\left(\mathrm{h}^{2} \mathrm{mf}\right)$ fue de 0,88 , por tanto, la ganancia genética respectiva con respecto al promedio del ensayo se estimó en un $16,5 \%$, hasta un $30,9 \%$ en relación con el material local o testigo.

La interacción genotipo-ambiente es un parámetro que registra el grado de adaptabilidad y comportamiento de los genotipos en varios ambientes. Por tanto, es esencial su análisis para reducir pérdidas en productividad cuando se busca plantar en todos los ambientes deseados, las colecciones de genotipos seleccionados (Zobel y Talbert 1988). Los valores de interacción genotipo-ambiente estimados en este estudio fueron casi nulos en $\operatorname{los} 8$ caracteres investigados $\left(\mathrm{c}^{2}\right.$ int $<0,04$, Cuadro 1$)$. La Figura 2 muestra claramente la existencia de 3 grupos de familias, las de alto, medio y bajo rendimiento. Puede también observarse que el grupo de las mejores 10 familias (con excepción de la familia M2) es prácticamente el mismo en ambos sitios, con ligeros cambios no significativos en el ranking genético. Así también ocurre con el grupo de las peores 10-12 familias, con excepción de la familia C26 que registra una caída importante y un pobre desempeño en el sitio San Carlos.

Con el objetivo de conformar un huerto semillero, se seleccionaron las mejores 12 familias del ranking genético del volumen comercial.ha ${ }^{-1}$. Pero para lograr una mayor ganancia genética, se utilizó una restricción adicional al elegir los 2 mejores individuos de cada una de estas 12 familias. Con esto se logra una conciliación entre un buen diferencial de selección y ganancia genética, con un buen tamaño efectivo poblacional (base genética y diversidad del programa). Una plantación establecida con semilla mejorada de

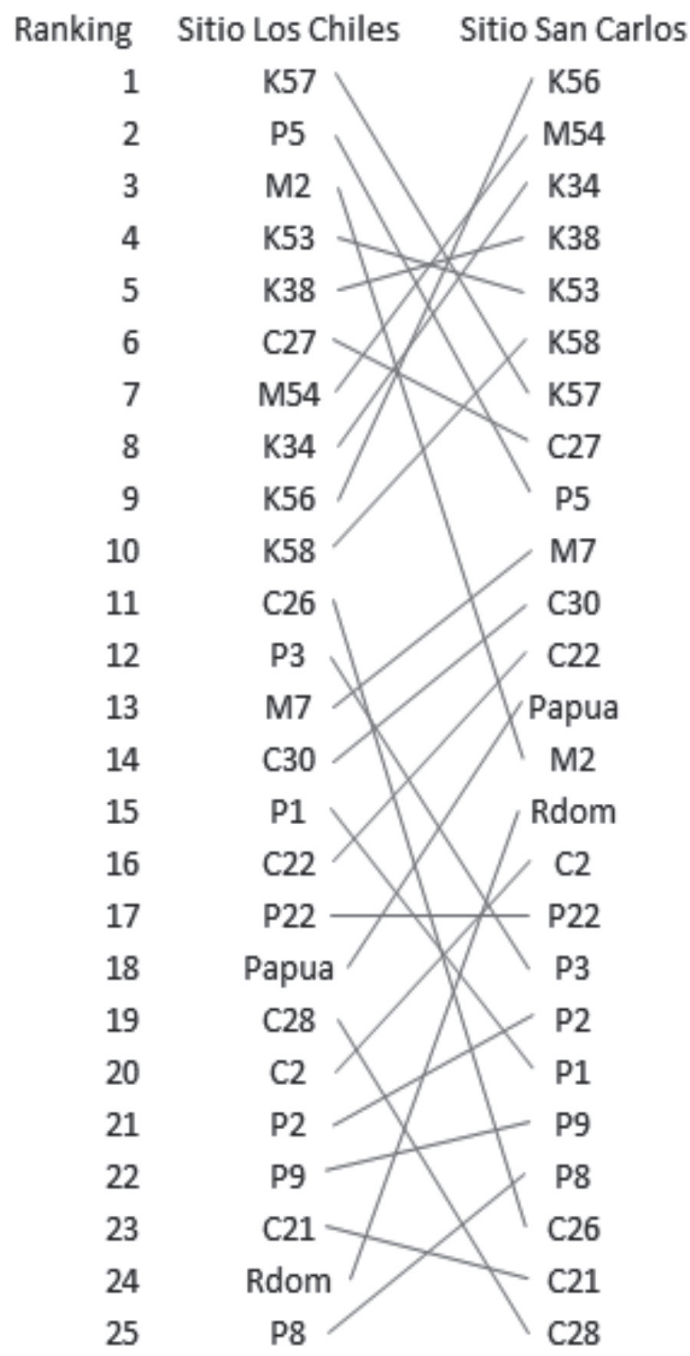

Fig. 2. Ranking genético de familias de A. mangium a los 4 años, en relación con el volumen comercial por hectárea, en la zona norte de Costa Rica.

un huerto semillero con estos 24 árboles tendría $78,93 \mathrm{~m}^{3} \cdot \mathrm{ha}^{-1}$ de madera comercial sin defectos y diámetro mínimo de $10 \mathrm{~cm}$ a los 4 años con una densidad inicial de 1111 árboles por hectárea (Cuadro 3). Este valor supera en $35,85 \mathrm{~m}^{3}(89,4 \%)$ al promedio individual poblacional. 
Cuadro 3. Ranking genético de los mejores individuos dentro de las mejores 12 familias de A. mangium a los 4 años, en relación con su volumen comercial por hectárea, en la zona norte de Costa Rica.

\begin{tabular}{|c|c|c|c|c|c|c|}
\hline Posición & Familia & $\mathrm{f}$ & $\mathrm{a}$ & Ganancia & $\begin{array}{c}\text { Nuevo } \\
\text { Promedio }\end{array}$ & $\mathrm{Ne}$ \\
\hline 1 & K57 & 145,082 & 56,366 & 56,366 & 99,45 & 1,00 \\
\hline 2 & M2 & 130,39 & 49,548 & 52,957 & 96,04 & 2,00 \\
\hline 3 & K53 & 131,335 & 47,845 & 51,253 & 94,33 & 3,00 \\
\hline 4 & K38 & 126,567 & 46,844 & 50,151 & 93,23 & 4,00 \\
\hline 5 & M2 & 123,979 & 43,800 & 48,520 & 91,60 & 4,71 \\
\hline 6 & P5 & 114,894 & 41,155 & 47,468 & 90,55 & 5,71 \\
\hline 7 & M54 & 119,168 & 40,640 & 46,615 & 89,69 & 6,72 \\
\hline 8 & $\mathrm{~K} 38$ & 109,7 & 39,649 & 45,841 & 88,92 & 7,38 \\
\hline 9 & M54 & 111,908 & 39,600 & 45,217 & 88,30 & 8,00 \\
\hline 10 & K57 & 109,474 & 39,496 & 44,697 & 87,78 & 8,57 \\
\hline 11 & K56 & 111,207 & 39,262 & 44,244 & 87,32 & 9,63 \\
\hline 12 & K56 & 105,172 & 39,139 & 43,851 & 86,93 & 10,18 \\
\hline 13 & K53 & 115,796 & 39,071 & 43,510 & 86,59 & 10,71 \\
\hline 14 & P5 & 112,562 & 37,813 & 43,130 & 86,21 & 11,20 \\
\hline 15 & K34 & 108,42 & 37,527 & 42,780 & 85,86 & 12,31 \\
\hline 16 & $\mathrm{C} 27$ & 112,648 & 37,499 & 42,469 & 85,55 & 13,40 \\
\hline 17 & $\mathrm{C} 27$ & 97,197 & 36,402 & 42,132 & 85,21 & 13,91 \\
\hline 18 & K34 & 95,397 & 35,921 & 41,805 & 84,88 & 14,40 \\
\hline 19 & $\mathrm{C} 26$ & 109,59 & 35,457 & 41,488 & 84,57 & 15,51 \\
\hline 20 & $\mathrm{C} 26$ & 100,216 & 31,216 & 40,999 & 84,08 & 16,00 \\
\hline 21 & P3 & 96,438 & 28,618 & 39,922 & 83,00 & 17,11 \\
\hline 22 & P3 & 90,112 & 28,092 & 39,429 & 82,51 & 17,60 \\
\hline 23 & K58 & 86,55 & 23,490 & 37,394 & 80,47 & 18,71 \\
\hline 24 & K58 & 77,672 & 20,882 & 35,850 & 78,93 & 19,20 \\
\hline
\end{tabular}

Donde:

f: valor fenotípico individual.

a: efecto genético aditivo predicho.

Nuevo valor promedio genotípico si se plantara solamente la semilla de este individuo y de todos los demás que lo superen en el ranking.

Ne: tamaño efectivo poblacional.

\section{DISCUSIÓN}

El crecimiento registrado en los materiales seleccionados puede ser considerado como muy bueno, con un IMA del DAP cercano a $4 \mathrm{~cm} . a^{-a n o}{ }^{-1}$, que supera en mucho a los $3 \mathrm{~cm} \cdot \mathrm{año}^{-1}$ propuestos para los mejores sitios de Costa Rica (CATIE 1992). El IMA del volumen comercial de casi $11 \mathrm{~m}^{3} \cdot \mathrm{ha}^{-1} \cdot \mathrm{año}^{-1}$ es sumamente alto a pesar de la corta edad del ensayo. En pocos años, este valor 
de productividad sin duda aumentará considerablemente, dado el efecto del diámetro y la altura comercial en el estimado del volumen comercial.

No hubo evidencia importante de una interacción genotipo ambiente $\left(\mathrm{c}^{2} \mathrm{int} \leq 0,04\right)$ entre ambos sitios en ninguno de los 8 caracteres investigados. Los mejores 9 genotipos se repiten en ambos sitios excepto la familia M2, que cambió de la posición 3 a la 14 (Figura 2). El grupo de las mejores 10 familias en volumen comercial, permaneció prácticamente unido, con ligeros cambios en el ranking genético. Así también, las 12 peores familias permanecieron unidas en ambos sitios, con muy ligeros cambios en el ranking con excepción de la familia C26, originaria de la zona norte de Costa Rica. Los valores bajos de la interacción genotipo $\mathrm{x}$ ambiente en todos $\operatorname{los} 8$ caracteres evaluados es alentador, ya que permite la utilización de las familias superiores en toda la zona norte de Costa Rica, y a la espera de resultados de productividad similares. La baja o casi nula interacción genotipo ambiente, simplifica en gran medida las siguientes etapas del programa de mejoramiento genético de la especie.

Las diferencias ambientales entre ambos sitios pueden considerarse relativamente leves y principalmente explicadas por variación en las características del suelo. El sitio Los Chiles está caracterizado por presencia de suelos poco profundos, mal drenados, propio de los sitios cercanos al gran lago de Nicaragua, frontera norte de Costa Rica. La distancia geográfica entre los 2 sitios es inferior a los $50 \mathrm{~km}$ y está conformada por una vasta planicie ondulada. Por lo tanto, las diferencias climáticas entre ambos sitios no se espera que lleguen a ser significativas.

\section{Correlaciones genéticas}

Se indica que las características genéticas que favorecen la calidad y buena forma del árbol van asociadas con características de crecimiento volumétrico lento. Estos datos sugieren que a mayor tasa de incremento en diámetro, la calidad del fuste muestra una tendencia a disminuir. La variable calidad está determinada en gran medida por ambas variables de bifurcación, por tanto, a mayor altura de bifurcación mejor es la calidad del árbol $(\mathrm{r}=0,87)$ y a mayor presencia de bifurcación peor es la calidad $(\mathrm{r}=-0,68)$.

El diámetro al año 1 estuvo fuertemente relacionado con el diámetro del año $4(\mathrm{r}=0,90)$. Por lo tanto, el potencial de crecimiento diamétrico que exhiben los árboles se expresa desde temprana edad y se ha mantenido de manera robusta hasta el año 4. Esto podría ser utilizado operativamente, dado que permitiría realizar selección temprana en esta especie. Esta correlación juvenil-adulto tan alta para el DAP, puede ser explicada en relación con la tasa de crecimiento tan acelerada de esta especie y a que la $A$. mangium es una especie de un ciclo de vida corto (se estima menor a 15-20 años, CATIE 1992).

La baja tasa de mortalidad registrada en el ensayo (8\%) pudo afectar los resultados de este parámetro. Debe recordarse que mortalidad puede registrar efectos confundidos y en ocasiones, de difícil separación entre una pobre adaptabilidad al sitio (información normalmente buscada) con el resultado de errores en el establecimiento y manejo del ensayo (Badilla et ál. 2002).

Finalmente se estimó una ganancia genética (GG) del $55,8 \%$ en la variable "volumen comercial" por hectárea, si se seleccionaran las 12 mejores familias y luego los 2 mejores árboles dentro de cada una de estas familias.

$\mathrm{GG}=\mathrm{S}$ entre familias $\mathrm{x} \mathrm{h}^{2} \mathrm{mf}+\mathrm{S}$ dentro de familias $\mathrm{x} \mathrm{h} \mathrm{h}^{2} \mathrm{ad}$

$\mathrm{GG}=\left(8,09 \mathrm{~m}^{3} \cdot \mathrm{ha}^{-1}\right) \times 0,88+\left(30,72 \mathrm{~m}^{3} \cdot \mathrm{ha}^{-1}\right) \times 0,55$

$\mathrm{GG}=7,12 \mathrm{~m}^{3} \cdot \mathrm{ha}^{-1}+16,9 \mathrm{~m}^{3} \cdot \mathrm{ha}^{-1}=24,02 \mathrm{~m}^{3} \cdot \mathrm{ha}^{-1}$ $=55,8 \%(62,9 \%$ con respecto al material local $)$.

\section{AGRADECIMIENTOS}

El trabajo formó parte de un proyecto de investigación financiado por la Vicerrectoría de Investigación y Extensión del Instituto Tecnológico de Costa Rica y empresas miembro de GENFORES (Cooperativa de mejoramiento genético de Costa Rica). 


\section{LITERATURA CITADA}

BADILLA Y., MURILLO O., OBANDO G. 2002. Efecto de la zona de vida y la altitud en la mortalidad y adaptabilidad al primer año de especies forestales en la Cordillera Volcánica Central, Costa Rica. Agronomía Costarricense 26(1):7-15.

BALIEIRO F DE C., FRANCO A.A., FONTES R.L.F., DIAS L.E., CAMPELLO E.F.C., DE FARIA S.M. 2007. Evaluation of the throughfall and stemflow nutrient contents in mixed and pure plantations of Acacia mangium, Pseudosamenea guachapele and Eucalyptus grandis. Revista Árvore 31(2):339-346.

BUEREN M. VAN 2004. Acacia hybrids in Vietnam: ACIAR Project FST/1986/030. ACIAR (Australian Centre for International Agricultural Research). Canberra, AU. 44 p.

CAPRONI A.L., FRANCO A.A., BERBARA R.L.L., GRANHA J.R.D. DE O., MARINHO N.F. 2005. Fungos micorrízicos arbusculares em estéril revegetado com Acacia mangium, após mineração de bauxita. Revista Árvore 29(3):373-381.

CATIE (CENTRO AGRONÓMICO TROPICAL DE INVESTIGACIÓN Y ENSEÑANZA). 1992. Mangium, Acacia mangium Will., especie de árbol de uso múltiple en América Central. Turrialba, Costa Rica. $62 \mathrm{p}$.

DE ASSIS I.R., DIAS L.E., ABRAHÃO W.A.P., RIBEIRO JR E.S., DE MELLO J.W.V. 2011. Camadas de cobertura para o crescimento de árvores e arbustos sobre um substrato sulfetado de mineração de ouro. Revista Árvore 35(4):941-947.

ESPITIA M., MURILLO O., CASTILLO C., ARAMÉNDIZ H., PATERNINA N. 2010. Ganancia genética esperada en la selección de acacia (Acacia mangium WILLD) en Córdoba (Colombia). Revista U.D.C.A Actualidad \& Divulgación Científica 13(2):99-107.

GUEVARA M., MURILLO O. 2009. Costos y rendimientos de ocho tipos de poda en plantaciones jóvenes de Acacia mangium Willd en la zona norte de Costa Rica. Kurú 6(17). 7 p.

KIM N., MATSUMURA J., ODA K., CUONG N. 2008 Possibility of improvement in fundamental properties of wood of acacia hybrids by artificial hybridization. Journal of Wood Science 55(1):8-12.

MESÉN J.F. 1990. Resultados de ensayos de procedencias en Costa Rica. Turrialba, Costa Rica. CATIE. Serie Técnica Informe Técnico, $N^{\circ} .146 .42$ p.

MURILLO O. 2011. Estrategia de mejoramiento genético para la cooperativa GENFORES. Ponencia magistral. In: XII Congreso Nacional Colombiano de Mejoramiento Genético de Cultivos. Montería, Córdoba, Colombia. 22-24 de junio, 2011.

MURILLO O., BADILLA Y. 2004a. Breeding teak in Costa Rica, pp. 105-110. In: B. LI and S. Mckeand (eds.).
IUFRO Meeting. Forest Genetics and Genomics. 1-5 de noviembre. Charleston, South Carolina, USA. Forest genetics and tree breeding in the age of genomics-progress and future. Raleigh, North Carolina State University. 2004.

MURILLO O., BADILLA Y. 2004b. Calidad y valoración de plantaciones forestales. Manual. Taller de Publicaciones del Instituto Tecnológico de Costa Rica. Escuela de Ingeniería Forestal. Cartago, Costa Rica. $51 \mathrm{p}$.

MURILLO O., BADILLA Y. 2006. Proyecciones de crecimiento de las plantaciones de Acacia mangium de ECOdirecta establecidas en la zona norte de Costa Rica. Informe de consultoría, FUNDATEC, octubre 2006. 27 p.

MURILLO O., BADILLA Y. 2011. Avalúos Forestales 2.0 (Software). Instituto Tecnólógico de Costa Rica. Cartago, Costa Rica.

MURILLO O., OBANDO G., BADILLA Y., ARAYA E. 2001. Estrategia de mejoramiento genético para el Programa de Conservación y Mejoramiento Genético de especies forestales del ITCR/ FUNDECOR, Costa Rica. Revista Forestal Latinoamericana 16(30):273-285.

NIRSATMANTO A., LEKSONO B., KURINOBU S., SHIRAISHI S. 2004. Realized genetic gain observed in second-generation seedling seed orchards of Acacia mangium in South Kalimantan, Indonesia. Journal of Forest Research 9(3):265-269.

PASTRANA I. 2011. Potencial genético de la selección de Acacia (Acacia mangium Willd.) en Córdoba (Colombia). Tesis M.Sc. Universidad de Córdoba. Facultad de Ciencias Agrícolas. Posgrado en Fitomejoramiento. Montería, Córdoba, Colombia. 112 p.

PASTRANA I., ESPITIA M., MURILLO O. 2012. Evaluación del potencial de mejoramiento genético en el crecimiento en altura de Acacia mangium Willd. Acta Agronómica 61(2):143-150.

PAVLOTZKY B., MURILLO O. 2012. Ganancia genética esperada en Acacia mangium en Los Chiles, Costa Rica. Agronomía Mesoamericana 23(1):1-13.

RESENDE M.D. 2002. Genética Biométrica e Estatística no Melhoramento de Plantas Perenes. EMBRAPA. Brasilia, Brasil. $975 \mathrm{p}$

RESENDE M.D. 2006. O Software Selegen-REML/BLUP. Manual. EMBRAPA. Campo Grande, Brasil. 299 p.

RESENDE M.D. 2007. SELEGEN-REML/BLUP Versión 2007. Sistema Estatístico e Seleção Genética Computadorizada (Software). EMBRAPA. Brasilia, Brasil.

SALAZAR R., MESEN J.F. 1990. Provenance analysis of Acacia mangium in Costa Rica. Presented at IX Forestry World Congress, Montreal, Canada, August 5-19. 
SIRZEE (SISTEMA DE INFORMACIÓN REGIONALZONA ECONÓMICA ESPECIAL). 2010. Mapas personalizados. San Carlos, CR, ITCR. Consultado 5 julio 2010. Disponible en http:// www.sirzee.itcr.ac.cr
STEEL R., TORRIE J. 1980. Principles and Procedures of Statistics. McGraw-Hill. New York. 633 p.

ZOBEL B., TALBERT J. 1988. Técnicas de mejoramiento genético de árboles forestales. México, D.F. México. Editorial LIMUSA. 546 p. 
